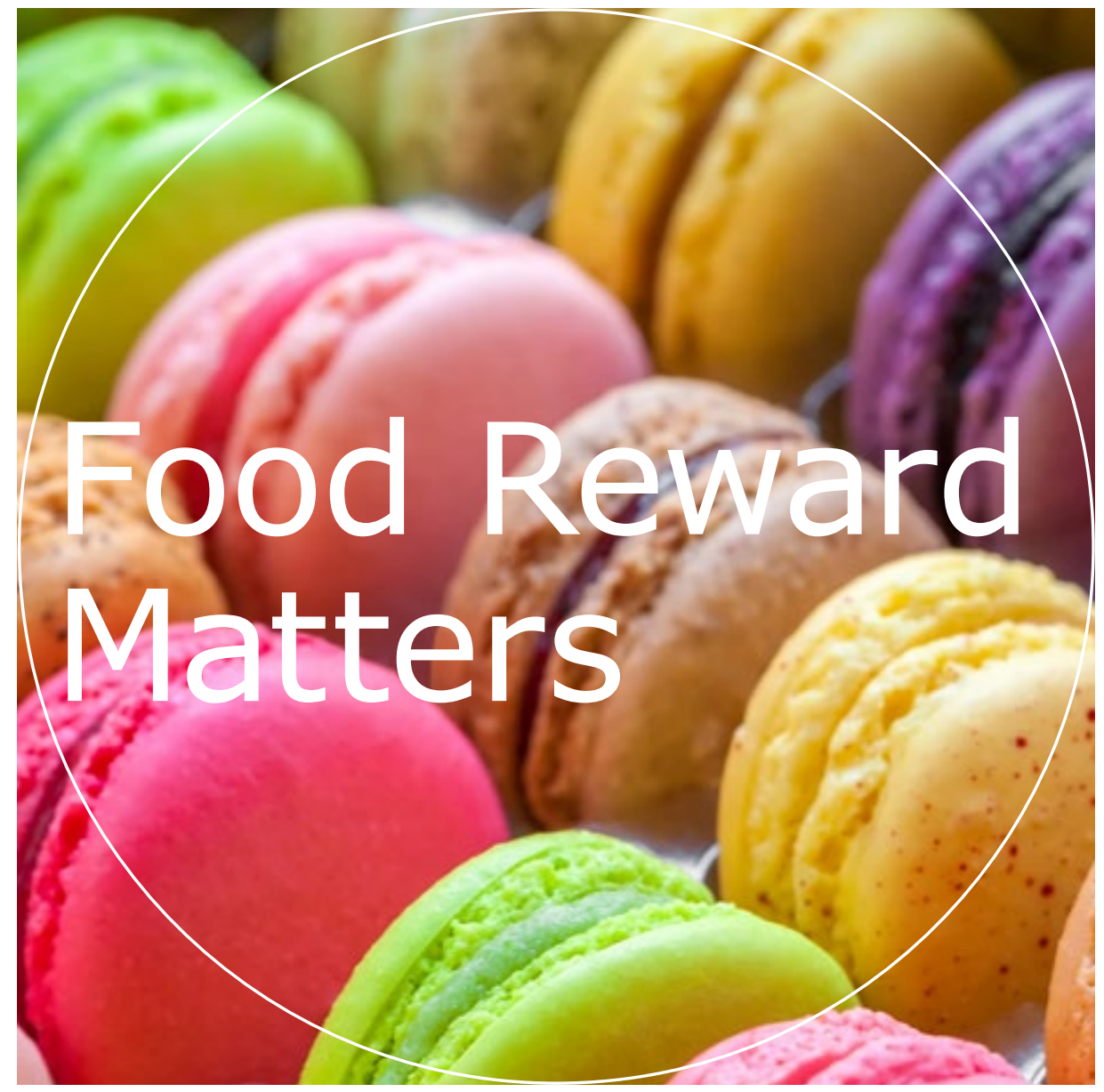

Prof. dr. Liesbeth Zandstra

Inaugural lecture upon taking up the position of Special Professor of Food Reward and Behaviour at Wageningen University \& Research on 11 October 2018 



\section{Food Reward Matters}

\section{Prof.dr. Liesbeth Zandstra}

Inaugural lecture upon taking up the position of Special Professor of Food Reward and Behaviour at Wageningen University \& Research on 11 October 2018 
DOI $10.18174 / 46455^{\circ}$

ISBN 978-94-6343-543-7 


\section{Food Reward Matters}

Esteemed Rector Magnificus, dear colleagues, students, family and friends,

\section{Introduction}

Have you ever had this experience that once you had opened a bag of M\&M's, you liked them so much --the crunchy candy shell with the sweet chocolate taste-- that before you knew it you had eaten the entire bag? Or that you unintentionally gave in to eating snacks -- like those Dutch snacks 'bitterballen'-- because of their great taste? The salty crispy coating with the thick, creamy meat paste inside? Food products can be really rewarding in that sense.

In this inaugural lecture, I want to explore matters of food reward and discuss why food rewards matter. My research explores matters of food reward in relation to health, as we still don't really understand why people choose and eat some food products and not others. My research also explores food rewards that matter, to try to understand how food rewards can support healthy eating behaviour.

In the next 45 minutes, I hope to convey to you some of the excitement I have for this topic and I will tell you the hopes and ambitions that I have for the future of research on food reward and behaviour. I will talk about the two research lines of my chair. First, I will discuss the impact of the dynamics of sensory perception and reward on repeated food choice and eating behaviour, and then I will elaborate on the measurement of sensory perception and reward in ecologically valid 'real-life' contexts.

Why is that so important? The worldwide prevalence of obesity has nearly doubled since $1980^{(1)}$. The top of the figure shows the prevalence of obesity in the United States over the last few decades. As you can see, there has been a dramatic increase in obesity over time. And this is a global issue. We see similar patterns all over the world, including Asia. Obesity is a concern because it is associated with serious health consequences such as cardiovascular diseases, diabetes, some types of cancer and reduced quality of life. 


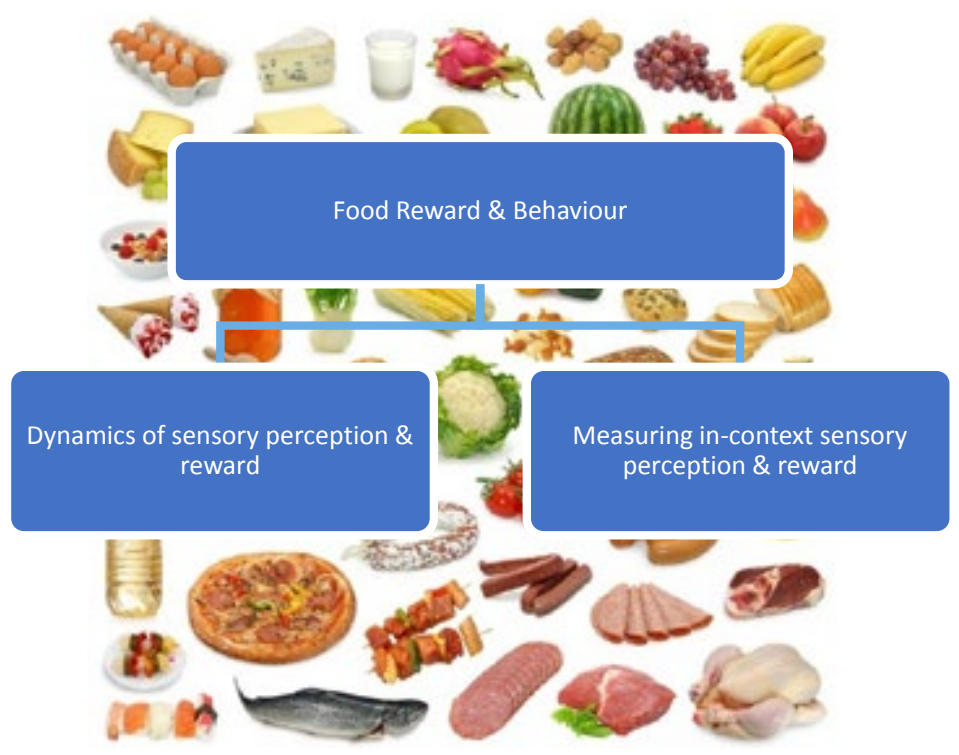

Figure 1. The two research lines of my chair Food Reward and Behaviour: 1) Dynamics of sensory perception and reward, and 2) Measuring in-context sensory perception and reward.
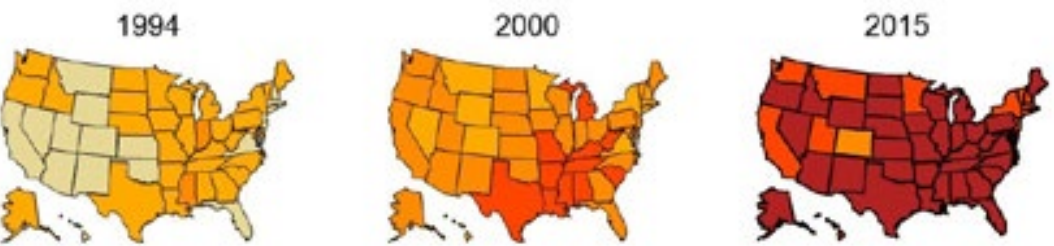

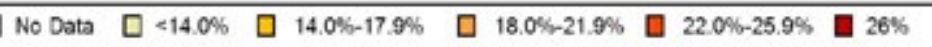

Figure 2. Prevalence of obesity in the United States over the last few decades (National Center for Chronic Disease Prevention and Health Promotion, Division of Nutrition, Physical Activity, and Obesity; https://www.cdc.gov/obesity/data/index.html).

It is therefore important to help people to change their eating behaviour to maintain a healthy weight and lower the risk of cardiovascular diseases, diabetes and cancer. For a healthy diet, most of us need to limit our intake of foods rich in fats, sugars and salt and increase the consumption of fruit and vegetables, in line with current dietary recommendations. And that is a challenge. Who doesn't love the taste of foods high in sugar, fat and salt, such as potato chips and cookies? They are rewarding! 
Consumer research consistently shows that taste is the key determinant that drives food choice ${ }^{(2)}$. With taste, I also mean the smell, appearance and texture of foods they are immediately rewarding. Health is only one of the many determinants that drives food choice, in addition to, for example, price, convenience and sustainability. The challenge here is that even though consumers know they need to eat healthily, they don't prioritize it at the point of purchase or consumption, as they don't perceive these effects as immediately and strongly as the taste of a product. Another challenge is that the failure rate for new products launched in the market is high: $80 \%$ of new food products fail within three years ${ }^{(3)}$. Many novel food products with initially high levels of reward seem to become 'boring' with extended use, leading to declining food acceptance and purchase frequency. Healthier alternatives of foods seem to be especially prone to this. To date, it is not clear how or why certain products become regularly consumed with a high repeat frequency over several years, and other products not.

That brings me to the overarching goal. We all know the aim of the World Health Organization to make the healthy choice the easy choice. I want to take this a step further: to make the healthy choice the preferred choice. How can we motivate people to select healthier alternatives, i.e., products that are reduced in sugar, fat and salt? And that they don't do this only once, but over and over again, so that it becomes a habit.

To make the healthy choice the preferred choice, it is essential to take a holistic approach by addressing three areas. Of course, we should focus on the product. But we should also focus on the consumer and the context, i.e., when you eat the product, where and with whom.

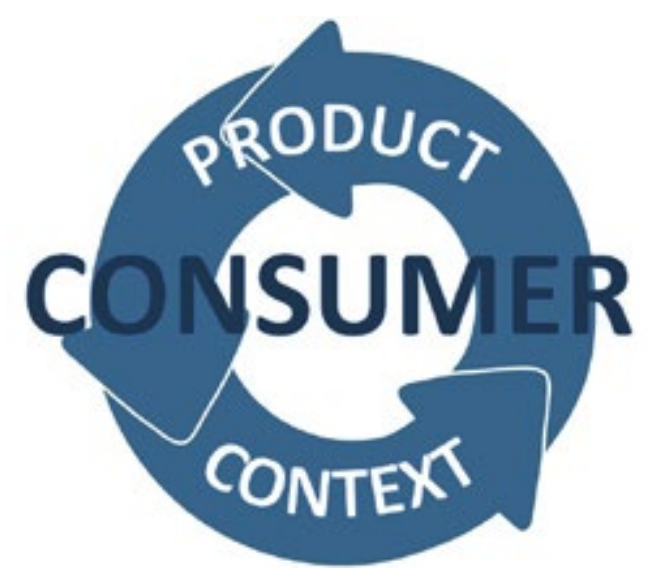

Figure 3. To make the healthy choice the preferred choice, we need to take a holistic approach: addressing the product, the consumer and the context. 


\section{Definition of reward}

Before I talk further about this, I would like to share my definition of reward and why I am interested in rewards. People engage in behaviours that are rewarding, that are beneficial to them. In my research, the term 'reward' has a broader definition than is customary in traditional research. I use it to mean any stimulus that generates positive, affective experiences, other than addictive food experiences (such as those delivered through alcohol and caffeine, for example). That is, the rewards or benefits from a product can be physiological, cognitive, emotional or social: a 'sweet taste', 'feeling relaxed', 'feeling full', 'doing good' - and so on. For example, evolution has designed us to find certain basic tastes inherently rewarding and others aversive. Give a baby a sweet taste on the tongue and it will respond with a happy face.

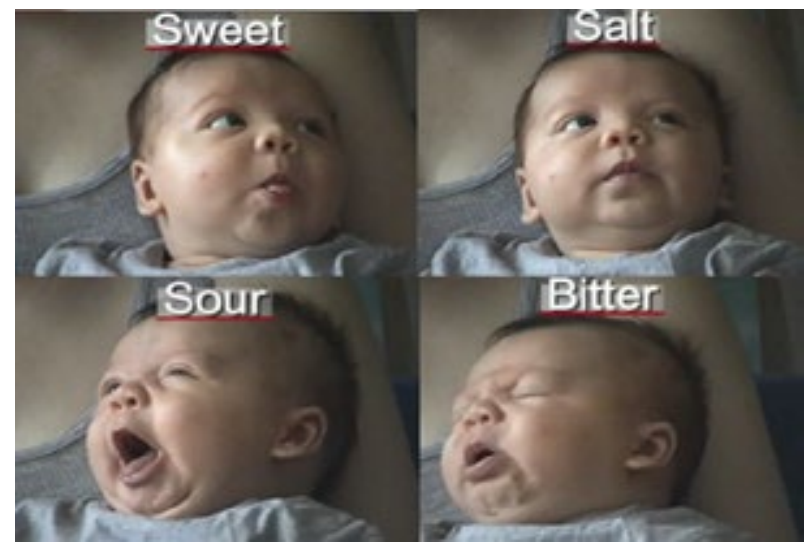

Figure 4. Facial expressions of a 15-month old baby when different solutions that varied in sugar, salt, sourness and bitterness were dropped on his tongue (with permission from Dr. Gie Liem).

Other tastes, though, either induce a neutral reaction (as with salt) or an adverse response (with sour and bitter). This is nicely shown by the facial expressions of baby Stijn who is shown on the slide: different solutions that varied in sugar, salt, sourness and bitterness were dropped on his tongue. From an evolutionary point of view, you could say that the sweet taste is a signal for calories, and that the bitter taste is a signal for danger, that it is potentially toxic or poisonous. With adults however, it is different. Their preferences have changed. Why? Because most food and drink preferences are learned. Throughout your whole life you come to learn to like new tastes and dislike other tastes. For example, coffee is bitter, which you don't like at birth, but really like as an adult. This has important potential implications in that if we understand more about how people learn to like new things, we can influence this through product development and communication strategies. 


\section{Dynamics of sensory perception and reward}

I will now focus on the direct interactions between the consumer and the product.

This relates to the first focus area of my research: the dynamics of sensory perception and reward. In a second step, I will talk about the consumer and the context. This is a relatively unexplored area and relates to my second research line on measuring in-context sensory perception and reward.

If I ask you the question: "Would you prefer $€_{5}$ now or $€_{10}$ in two weeks' time?", what would your answer be? Research suggests the former, the $€_{5}$, as people are often driven by immediate gratification, i.e., we tend to prefer immediate rewards over delayed rewards. A well-known experiment, the Stanford marshmallow test, shows the willpower needed to resist a short-term reward ${ }^{(4)}$. In this test, a child is sitting in a room with a marshmallow in front of him/her. A researcher gives the child a choice: "I will be leaving the room for a couple of minutes. You can eat the marshmallow immediately or wait until I return. You will then get an extra marshmallow". So, he/she then gets the two marshmallows instead of one, clearly a larger, albeit delayed, reward. As the marshmallow is so delicious, children find it difficult to resist and struggle with waiting. The kids that were able to wait for the extra marshmallow had all kinds of techniques to distract themselves such as turning around so that they couldn't see the marshmallow, stroking the marshmallow as if it were a tiny animal, or singing songs and clapping in their hands. Others immediately caved in and started eating the marshmallow as soon as the researcher left.

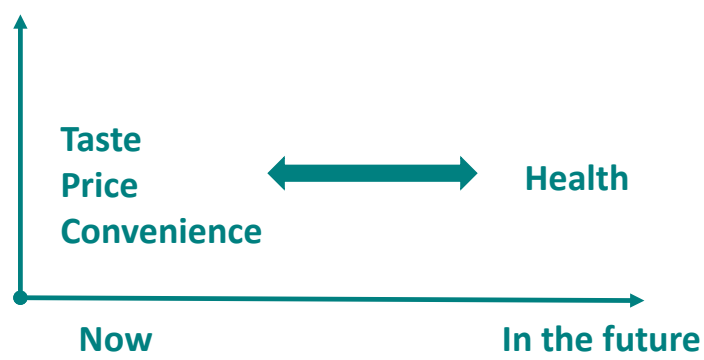

Figure 5. Immediate rewards are delivered directly at the point of consumption (e.g., taste, price), whereas delayed rewards are characterised by a delay, that is, it takes time before you perceive them (e.g., health).

In relation to health, we also talk about immediate rewards and delayed rewards. Examples of immediate rewards are the taste of the product, price, and convenience - they are experienced immediately, and they are rewarding. Examples of delayed rewards are long-term health benefits such as heart health or the prevention of dental caries. These rewards are delayed in time as it can take weeks, months or even years 
before you perceive them. How can we motivate people to resist the 'now' and invest in their future, leading to healthier habits? The challenge is to design healthier, yet immediately rewarding, alternative foods to ensure consumers will continue to like (or even prefer) these reformulated products. For example, they might include food products that are reduced in energy, saturated fat, added sugar and/or salt. However, we can only go so far in reducing fat, sugars and salt, because these are rewarding ingredients. The challenge lies in trying to find ways to compensate for the fact that, over time, liking will diminish as these ingredients are reduced. Recent developments in research suggest a degree of interchangeability of rewards that we might be able to exploit ${ }^{(5)}$. Natural rewards such as sugar or salt appear to be interchangeable with convenience as a rewarding experience in the preparation or eating of foods, or whether an individual receives an artificial reward such as money. If we remove one type of reward - fat for example - we might be able to replace it with another rewarding attribute, e.g., in the product, the communication or context, to ensure continued acceptance. For this, we need to build a fundamental understanding of the underlying mechanisms of reward to create healthy product experiences that evoke positive rewarding responses.

My first research line will focus on the dynamics of sensory perception and reward, and how they are learned and influenced. In presentations and lectures I always use the following example to illustrate this phenomenon. I ask people to think about a product that they really enjoy and eat over and over again. Everybody has their own favorite products, varying from chocolate, to coffee, cashew nuts, cheese, pizza etc., products coming from both the sweet and the savory food domain. Please try to think of an example for you personally as well.

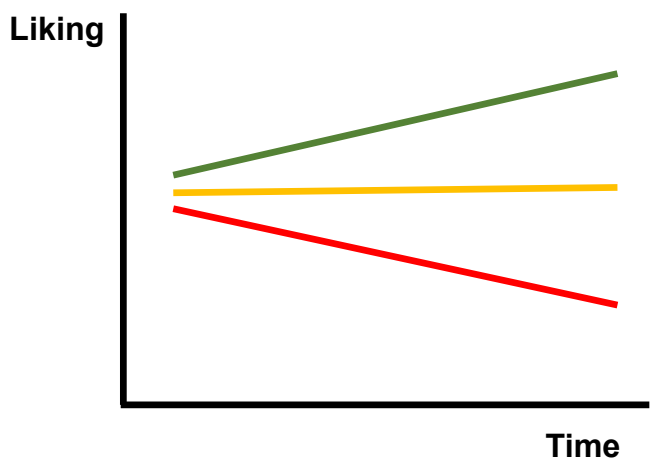

Figure 6. Liking is a dynamic process. It can increase, remain stable or decrease over repeated consumption. 
Next, please try to think about a product that you used to really enjoy, but now no longer do because it doesn't taste as good as it used to. Do you already have a product in mind? This question might be more challenging to answer, but here, again, people can name a variety of food products, coming from both the sweet as well as the savory ends of the food domain.

Liking is a dynamic process ${ }^{(6)}$. It can increase, remain stable or decrease over repeated consumption. When a novel product is launched on the market it elicits in consumers a high level of reward, which can attenuate over time as boredom sets in.

Staple foods, such as crackers, milk or bread on the other hand seem to be resistant to boredom as their liking ratings remain stable over repeated consumption.

How do these changes in liking come about? As I mentioned earlier, we know that liking is to a large extent learned and that there is a reward-based mechanism underlying it ${ }^{(7)}$. This is called associative conditioning, also known as Pavlovian conditioning in which the dog starts to salivate in response to a bell because of the learned association between the bell and the delivery of food. Here, the repeated consumption of a food product leads to a similar learned response that results in an increase or decrease in liking over time.

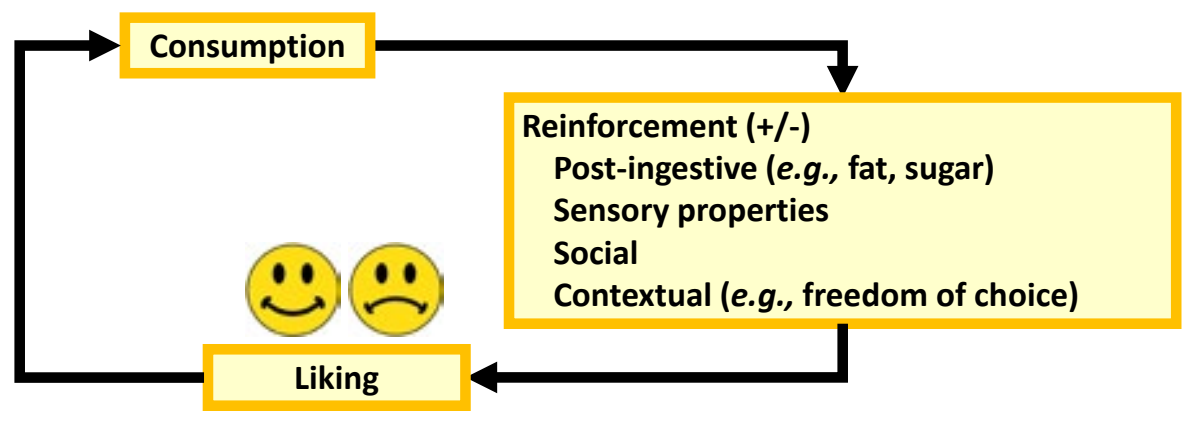

Figure 7. Liking is to a large extent learned.

The associative effect for which we have the most evidence is taste aversion ${ }^{(8)}$. A flavour that is associated with something that produces a negative post-ingestive effect such as nausea or sickness comes to be disliked. For example, a person who becomes very sick after eating shrimps may then become averse to the taste of shrimps, even though the sickness was merely coincidental and not related to the shrimps. This learned taste aversion usually requires only one exposure to the food, 
and, as a result, the food may be avoided for many years. In a similar manner, consumers learn to like foods that are associated with positive post-ingestive effects $^{(9)}$. For example, eating a product that is high in fat or sugar makes you feel comfortably full afterwards. This is a positive feeling. Over repeated consumption you then learn to associate this reinforcing positive feeling with the sensory properties of the food product which makes you like that product even more in the end; an increase in liking. This learned preference usually requires 3 to 15 exposures to the food, and as examples, you can think of products such as pizza, French fries and pancakes. Unfortunately, healthier alternatives of products lack the reinforcing post-ingestive effects and we therefore need to explore other ways to reinforce the experience, for example, via the social context or physical environment ${ }^{(10)}$.

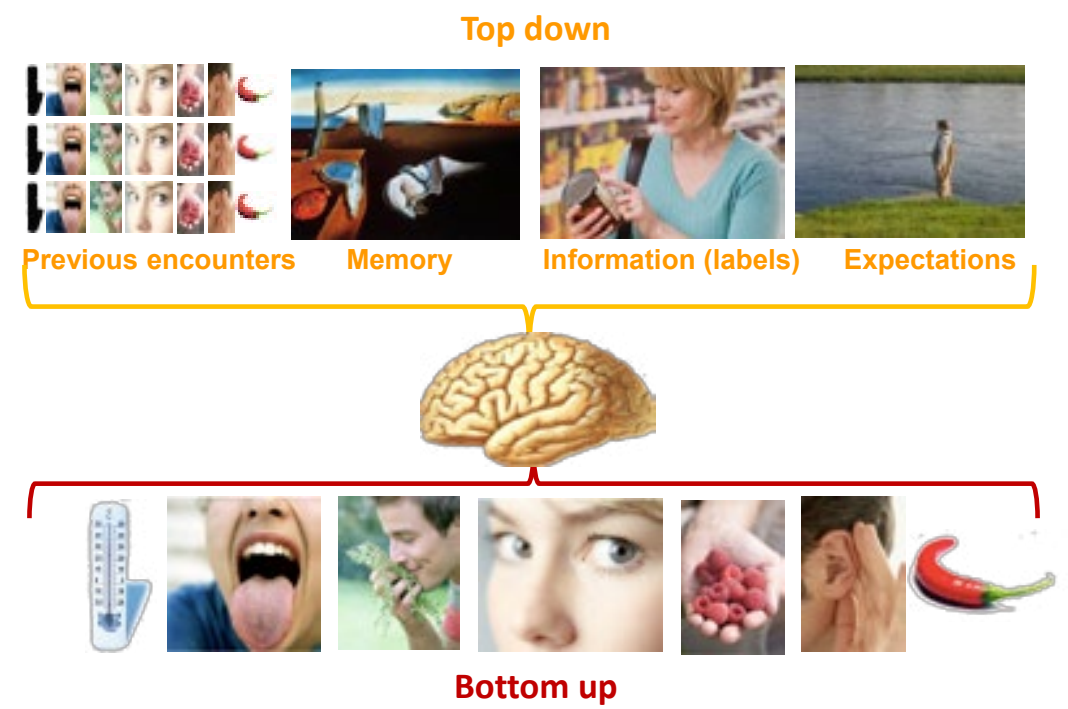

Figure 8. Bottom-up effects and top-down effects play a role in food perception and evaluation, based on Dijksterhuis (2017).

One way to look at this is by making a distinction between so-called bottom-up and top-down effects ${ }^{(11)}$. The bottom-up effects are the sensory systems as illustrated in the figure at the bottom (from left to right: temperature, taste, smell, vision, touch, audition and pain). When we perceive foods, many of these sensory systems are activated at the same time: our perception of the food we eat comes not only from what it looks, smells, and tastes like, but also from what it feels and sounds like in the mouth. At the top of the figure, cognitive top-down effects on taste and liking are 
shown. Some of these stem from previous encounters of a product, which reside in our memory. Another important influencer is cognitive information, which we get from advertisements, labelling on products or otherwise, and together these create expectations that drive our perception and liking of a product.

Top-down effects play an important role in food evaluation. Sometimes top-down effects are strong enough to override bottom-up effects such as sensory stimulation and they potentially drive choice behaviour. For example, a key experiment showed that people perceived an odour differently based on the word that was presented together with the smell ${ }^{(12)}$. Researchers asked participants to sniff the scent of the rather pungent odour isovaleric acid in combination with two different cognitive labels, either 'cheddar cheese' or 'body odour', and asked them to rate the experience. When the odour was labelled 'cheddar cheese', subjects liked the odour more than when it was called 'body odour', even though the odours were identical. Interestingly, the researchers also scanned the participants' brains using fMRI and found that these cognitive labels resulted in different activations deep down in the brain.

Similar cognitive effects and mechanisms have been found for the taste of wine, just to give you an example that is familiar to all of us ${ }^{(13)}$. If a person is told that he or she is tasting two different wines - and told that one costs $€_{5}$ and the other $€_{45}$, when they are, in fact, the same wine - the part of the brain that experiences pleasure will become more active when the drinker thinks he or she is enjoying the more expensive wine. There are limits to these effects, however. If you buy a bottle of good wine and find the taste to be only moderately fine, your preconditioned, internal representations of a good wine take over and you would still perceive it to taste pretty good. You will not do so if the so-called 'good' wine tastes like vinegar. In that case, the promise of a good product when the quality is poor leads to a decrease, instead of an increase, in liking. This is called the 'contrast' effect. These are just two examples to illustrate that cognitive top-down inputs can have profound effects on how we perceive the sensory and hedonic properties of foods, and that these can even influence neural responses in taste and reward-processing regions of the brain.

We know very little about how these top-down influences impact food choice behavior over time. We carried out a study in which a group of people were given a low- and high-energy yoghurt drink every day for 2 weeks $^{(14)}$. One day they got a high-energy drink, the other day they got a low-energy drink. Both drinks were similar in appearance, taste and texture, and subjects liked them equally. People were not aware that the drinks differed in any way, except that one drink was paired with a blue label, and the other with a pink label, as is shown on the slide. 


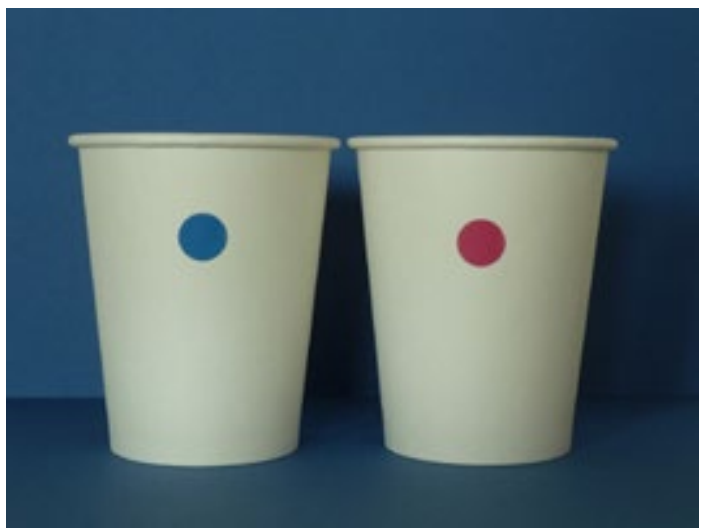

Figure 9. Consumers may still like the healthier alternative, but not choose it...

After 2 weeks, we asked them to make a choice which yoghurt drink they preferred. Although subjects were unaware of the different energy content of the drinks, they chose the 'high energy' drink more often and reported that they 'felt better and fuller' afterwards. This suggests a conditioned preference for a delayed physiological (energy) reward. We clearly need to find other ways to compensate for a loss in reward when we reduce energy levels in a product. It also suggests that this simple labelling seems to act as a trigger in the brain for choice and preference. When doing consumer research, it is therefore important to reflect and decide on what type of information is provided about the product to be tested. For example, will it be tasted blind, with a concept or branded?

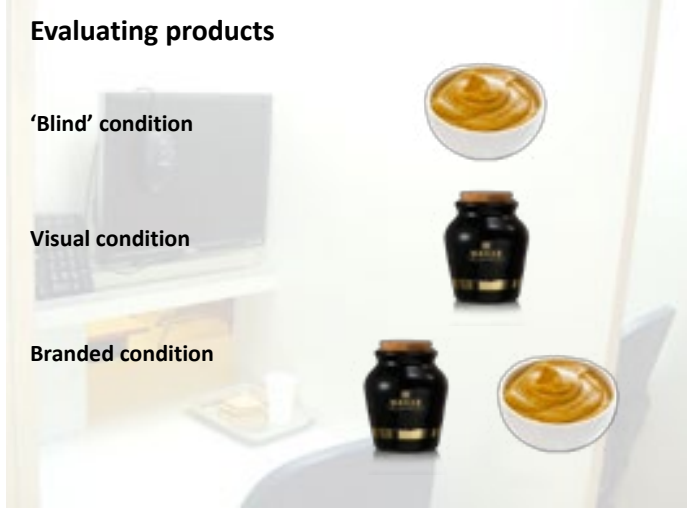

Figure 10. 'Blind' condition, visual condition and branded condition when evaluating products. 
1) In case of the 'blind' testing condition, consumers receive the product without knowing the product's brand. In this bottom-up approach, they simply taste the food and evaluate it solely on the basis of sensory characteristics. In this case a plain mustard. The advantage is that it is a clear evaluation of the product, without any influence or bias from the brand.

2) In the 'visual' condition, participants do not taste the product but asses the level of satisfaction on the basis of written and/or visual information. For example, a picture of a brand as is shown on the slide.

3) In the 'branded' condition, participants taste and evaluate the product together with written and/or visual information. Participants evaluate the mustard knowing which brand it is. The product's score is then affected by top-down influences of the brand, but the advantage is that you get an understanding on whether the product and brand fit together or not.

Recently, my PhD student, Irene Tijssen, who just successfully defended her thesis, investigated interactions between bottom-up and top-down influences in branded testing conditions. This included exploring the impact of the colour of packaging on the consumption experience of healthier foods ${ }^{(15)}$. In supermarkets, healthier foods are typically packaged in less vibrantly coloured packages compared to their regular counterparts, as is pointed out with the red arrow on the slide. If you didn't know this yet, just have a look at the products in the aisles the next time you are in the supermarket. The question we asked ourselves is: does this way of colouring communicate the intended message of a healthy, yet tasty and rewarding product? For a low-fat sausage and an energy-reduced dairy drink, we systematically altered three dimensions of colour on the packaging: 1 ) the hue (i.e., broad colour category, such as red and blue), 2) brightness (i.e., the amount of black/white added to the hue), and 3) saturation (i.e., the intensity of the hue). 

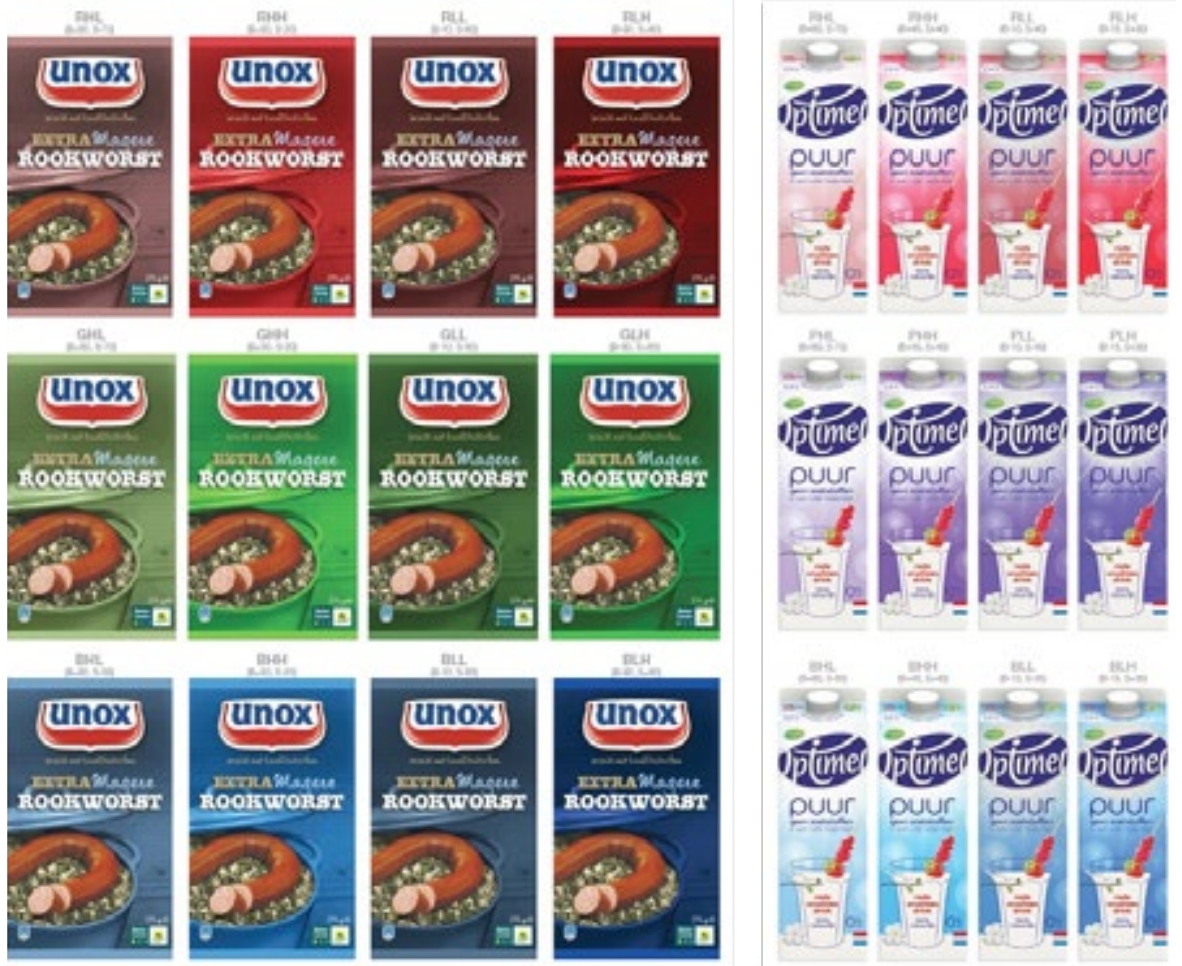

Figure 11. Packages varying in hue (i.e., broad colour category, such as red and blue), brightness (i.e., the amount of black/white added to the hue), and 3) saturation (i.e., the intensity of the hue) (based on Tijssen et al., 2017).

Vibrant packages, that is with increased saturation and decreased colour brightness, appeared to be most attractive and gave rise to expectations of a sweeter, creamiertasting and more intensely-flavoured product. Packaging with decreased saturation and increased brightness elicited healthier but less appealing expectations and this is actually what is currently broadly implemented in the supermarket. It may therefore be better to make the packaging colors of healthier products warmer, less bright and more saturated - that is to say, more similar to regular products to make them more flavourful, more attractive and more rewarding. What is undoubtedly new about this research, is that we explored subtle contrasts in very realistic and well-controlled stimuli, which means that we are making comparisons that map realistically to how consumers respond to products in daily life.

Moving forward, the challenge ahead is to bridge the gap between immediate and 
delayed rewards. A key area that warrants further research is how reward systems and expectations influence sensory perception and acceptance, not only initially, but also over repeated consumption. In the case of learned food-reward associations, we know very little about the stability of food-reward over repeated consumption. We need to find out how long it takes for a reward to fade out, trace more closely how top-down influences impact the reward experience and how the impact of these top-down influences evolve over time. Obviously, my aim is to boost and maintain the immediately perceived reward value over repeated consumption. Ultimately, future research should incorporate influences on sensory perception. This is from understanding the product composition ('bottom up'), the consumer and the environment ('top down') in an integrated way before, during and after consumption. In particular this will help to identify those characteristics of foods that increase their immediately perceived reward value. This will also enable new opportunities for creating innovative and healthy products. Next year, I hope that a post-doc will start working in this exciting area in a joint project with EWHA University in South-Korea. And I am looking forward to setting up more projects and initiatives in this promising area together with my colleagues at Wageningen University.

I now would like to continue with my second research line on measuring in-context sensory perception and reward, with a specific focus on the direct interaction between the consumer and the context.

\section{Measuring in-context sensory perception and reward}

What is the role of context in relation to food? The context in which food is selected and consumed is an important factor in its acceptability, choice and consumption. In the book 'The Psychology of Food Choice', Dr. Herb Meiselman writes in his chapter that 'The state of both the external environment and the individual prior to and during the eating experience is important ${ }^{\prime}{ }^{(16)}$. In my research, I address both the external environment, which is the physical context: where you eat, when you eat and with whom you eat, and the individual, which is the internal context, in which I specifically focus on the emotional state.

\section{Physical context: bringing the world into the lab}

I will first talk about the physical context. Research has shown that products are experienced differently in different physical contexts. For example, in one study, a dish, Chicken à la King and Rice, prepared with identical ingredients and to a standard recipe, was served to consumers in a variety of settings ${ }^{(17)}$. These ranged from a residential home for the elderly to a 4 -star restaurant. The results showed that the physical context contributed significantly to overall liking. A hierarchy of 
contexts emerged with upscale restaurants receiving higher scores than institutional settings. There is even a one scale point difference on a 9-point hedonic scale between the highest ratings of the dish in the 4-star restaurant, and the lowest ratings in an army training camp. It all relates to what I mentioned earlier: the top-down effects generate specific expectations. That is, food from a 4-star restaurant must be really good, which impacts on how the product is perceived and to what extent you like the product.

And you may be familiar with this effect yourself. Have you tasted a new drink on your holidays that you liked so much that you brought a bottle back home with you? But somehow at home it just didn't taste the same? This is all because of the different contexts. You need the atmosphere, heat and ambience of a relaxing holiday to really enjoy it.

Why is this phenomenon so important for my research? In traditional product tests, panelists evaluate products in isolated sensory booths where everything is as standardized as possible (i.e., temperature, light conditions, sound etc.) and non-product contextual information is intentionally minimized. This is completely different from real-life situations in which you drink or eat a product together with other people.

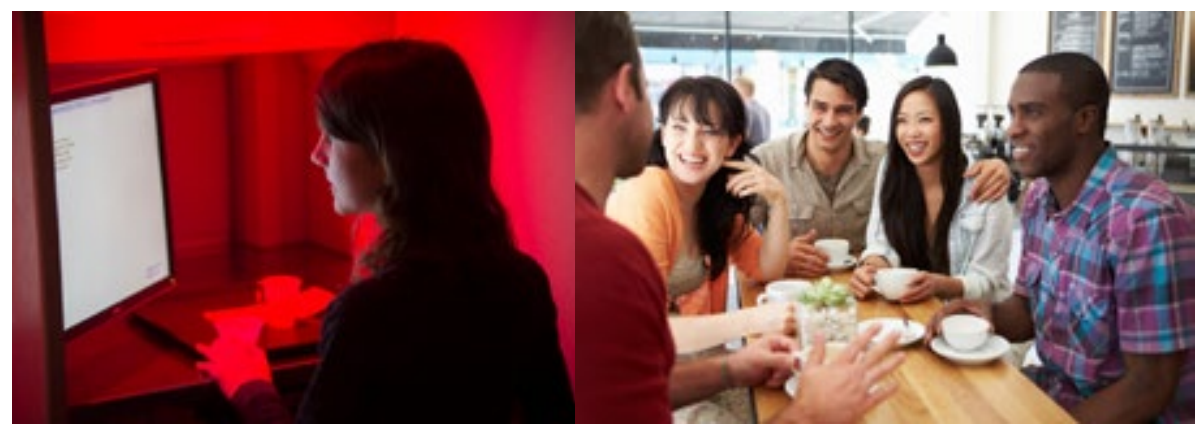

Figure 12. From lab to real-life contexts.

Whereas sensory booths enable a strict control over product testing, they are not representative of what happens in the real world and do not take the role of context in shaping product perceptions and acceptance into account. I therefore recommend to assess foods in realistic contexts to increase the external validity of the results of such tests. However, from a practical point of view, testing in real-life, such as at home, can be time consuming and expensive compared to traditional laboratory product tests. 
Furthermore, a downside is that there is very little control on how the product is being tested. For example, the experimenters do not know who really tasted and rated the product and under what circumstances. A solution would be to simulate the context in the laboratory, which would combine the increased experimental control of the laboratory with the increased realism of the simulated context.

A new and promising development to create simulated contexts is to use immersive technologies in sensory and consumer testing to improve the predictive validity and reliability of sensory and consumer testing. Immersive technologies are technologies that blur the line between the physical world and a digital or simulated world, creating a full sense of immersion. I am specifically interested in immersive technologies and not in virtual reality technologies at this stage. Virtual reality technologies offer ways to interact with virtual products in a virtual world with the use of headsets, but these headsets hinder the actual tasting and experience of real products, which is the focus of my research.
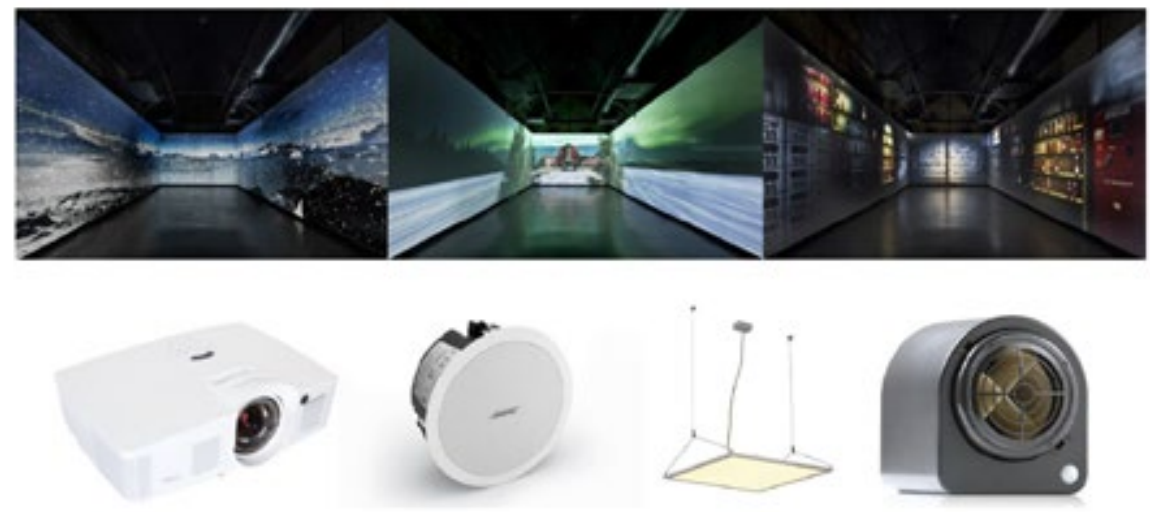

Figure 13. Momentum for immersive technologies.

Initial studies in the area of immersive technologies indicate that they add value to sensory and consumer testing. When people evaluated cookies in a traditional laboratory setting and an immersive setting using immersive technologies, the immersive setting generated more reliable data over time and people were more engaged and liked the cookies better than in the traditional testing context ${ }^{(18)}$. You can see on the slide that in the immersive setting, a video was shown of a man in the act of making cookies in a kitchen, and a cookie aroma was dispersed to fill the room with a subtle but noticeable aroma. We are at the beginning of research in this intriguing area. How can we best utilize emerging immersive technologies to 
create contextually-relevant virtual environments for the development of novel sensory and consumer-testing methodologies? Similarly, the use of immersive technologies provides the opportunity to investigate the role of visual, auditory and olfactory cues independently or when combined (as normally experienced in real-life settings) on sensory perception and liking in semi-real-life settings. We just started a project 'Breaking habits for the better', which is supported by the Dutch Top Consortium for Knowledge and Innovation (TKI) Agri\&Food. The aim of the project is to validate the use of immersive technologies to better understand food intensity and reward in different contexts. The project will be run together with Wageningen Food \& Biobased Research, Kikkoman Europe R\&D Laboratory, Unilever R\&D Vlaardingen and Noldus Information Technology. In this research, we make use of the newly developed immersive 'experience' room in the Helix Building at Wageningen University. Next year I hope to share the first results.

\section{Internal context: emotional state}

Food is emotion. The second part of my research line therefore focusses on the development of better tools to measure emotions evoked by foods, with a specific focus on measuring it in an implicit manner. That is to say, without asking consumers about their experiences. Questionnaires and rating scales are the most used measurement techniques in consumer research in which consumers are asked to verbally express responses to product experiences. This way of testing reflects conscious processing whereas most perceptions and consumer decisions are based on unconscious processes. Just like this iceberg.
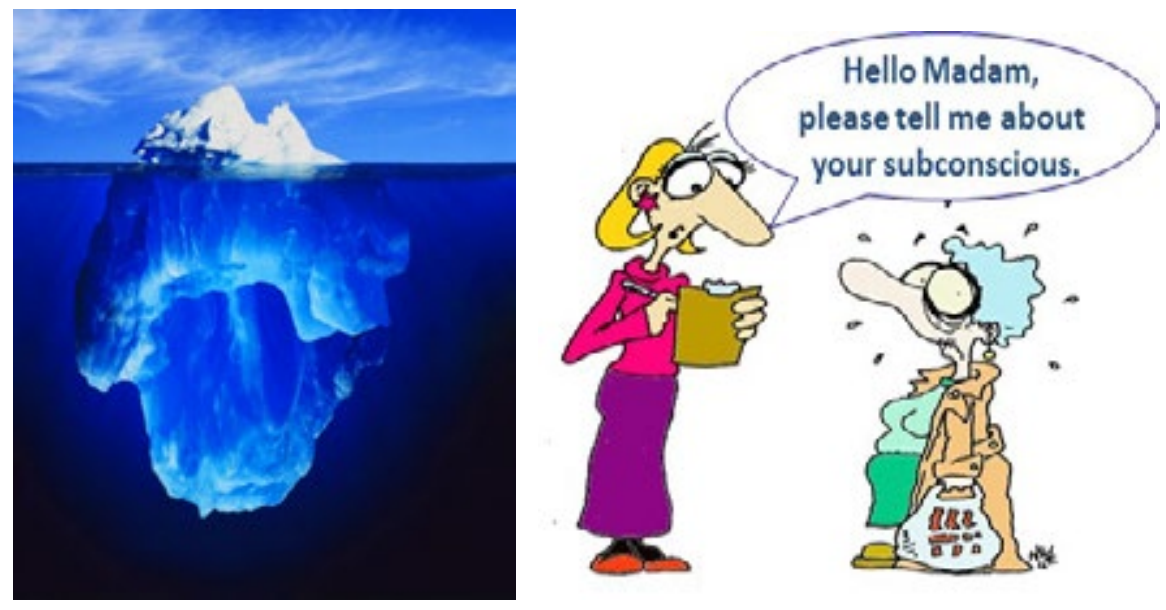

Figure 14. Measuring consumers' experiences without asking. 
The conscious mind is the small top of the iceberg above the water containing what you are aware of in your thoughts and actions. The unconscious part is the large bit beneath the surface, containing all the processing behind why you do, think, act, behave, and feel the way that you do. The majority of information that you receive from the outside world is automatically processed outside conscious awareness. Only a selection of this information 'pops' into consciousness. Asking consumers what they perceive and how they feel can therefore be of limited value when the 'true' answers are often driven by unconscious attitudes and emotions, rather than conscious thought. Implicit measures may therefore be better able to capture the perceptual experiences and emotional impact of products.

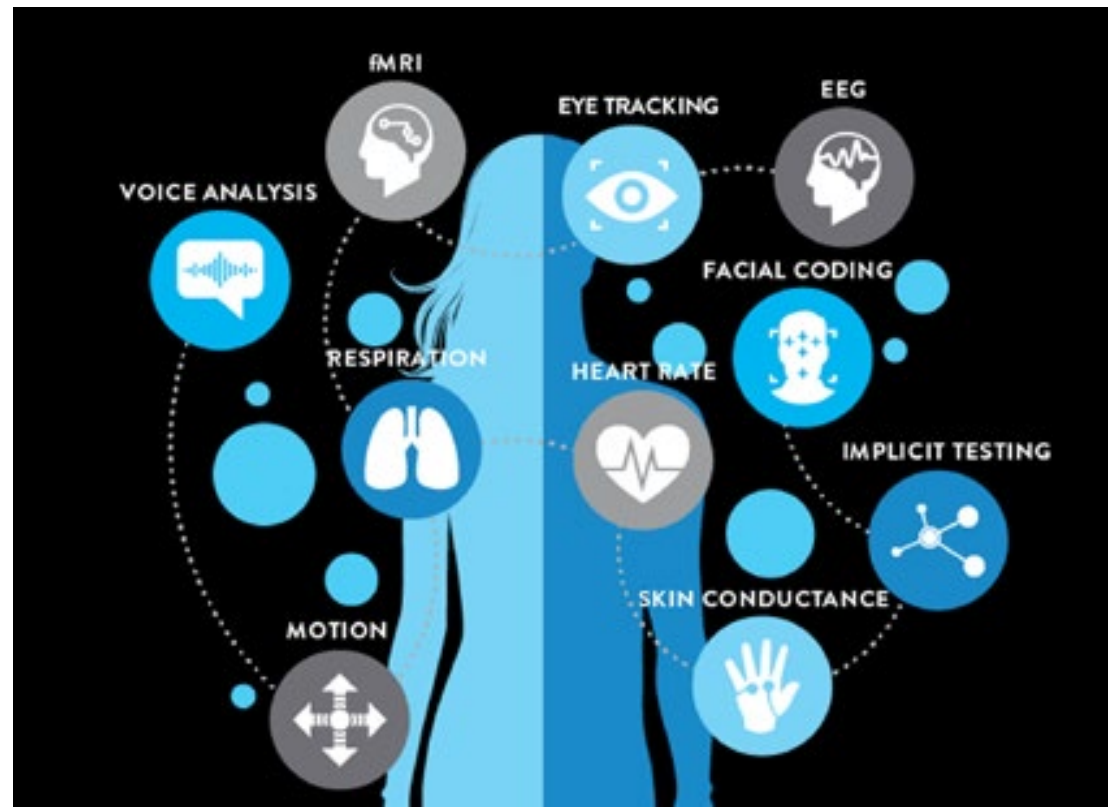

Figure 15. Implicit measures may be better able to capture the perceptual experiences and emotional impact of products.

Recent advances in implicit behavioural and neurophysiological measures (e.g., heart rate, skin conductance, EEG) are leading to new insights into brain functions and their relations with sensory perception, reward, cognition, emotions and behaviour. Implicit neurophysiological measures as such are not new. But the exploration and implementation of implicit measures in real-life contexts in the food domain is new and may provide a more fundamental understanding of how consumers process and respond to certain stimuli and what they really like over repeated consumption. 
We adopt the circumplex model of emotions from James Russell ${ }^{(19)}$, which describes emotions along two dimensions: valence and arousal. Valence, or pleasantness, so to say, represents the horizontal axis of the figure and arousal, or intensity, represents the vertical axis. Emotions can fall within the four arousal-valence categories.

\section{AROUSAL}

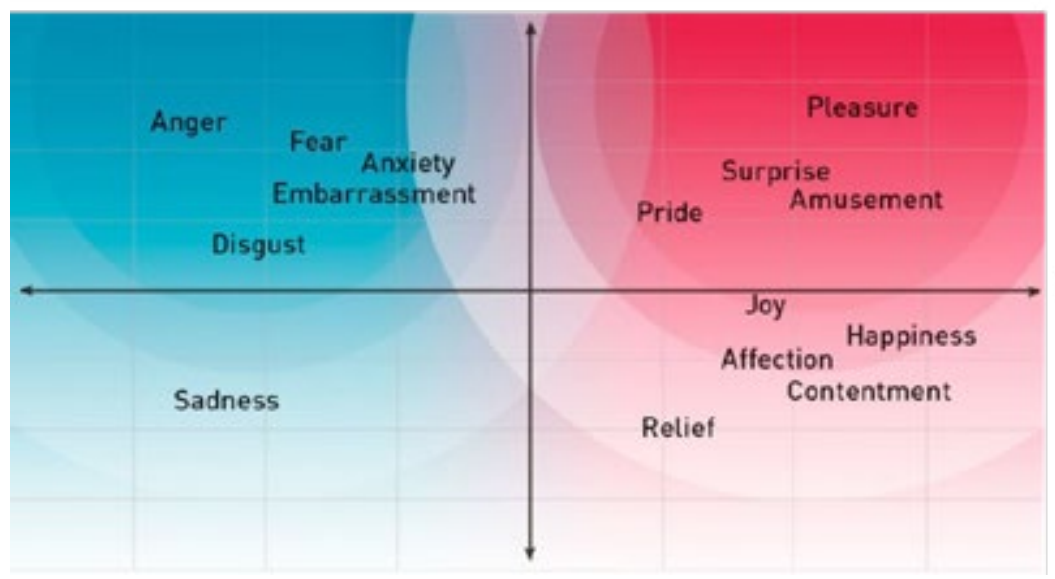

VALENCE

Figure 16. Circumplex model of emotions (Russell, 1980).

For example, if a product is high in arousal and high in valence it is experienced as positively pleasant or surprising, whereas if a product is high in arousal and low in valence, it can be experienced as disgusting. If we want to know people's emotions, we can simply ask them, and there are indeed explicit questionnaires and scales that assess emotions by the perceived degree of valence and arousal. However, as I just mentioned, self-report questionnaires and scales will only include conscious experiences that the individual chooses to share, and these scales can only be scored a few times by participants and at fixed moments in time. We are therefore working towards the capture of emotions via implicit methods since they do not interfere with the experience itself and are of a continuous nature. Brain signals that are measured with EEG may provide us with information about valence, as recent research showed, that positive and negative valence corresponded to different brain activations. Arousal is less clearly linked to brain activation, but can be measured peripherally through, for instance, skin conductance, pupil size and heart rate variability. 
We are now beginning to study implicit ways of testing to monitor experiences in real time, as it happens, in real-life food contexts and how it can complement our comprehensive range of more traditional consumer research measures. For example, in a project supported by the Dutch Top Consortium for Knowledge and Innovation (TKI) Agri\&Food, TNO, Unilever R\&D Vlaardingen, Eaglescience Software and Noldus Information Technology, we examined emotions in a cooking context by using EEG, heart rate variability and skin conductance. In a first study, participants prepared and tasted two stir-fry dishes ${ }^{(20)}$. The only difference between these dishes was the main ingredient: either chicken (the left photo) or mealworms (the right photo).

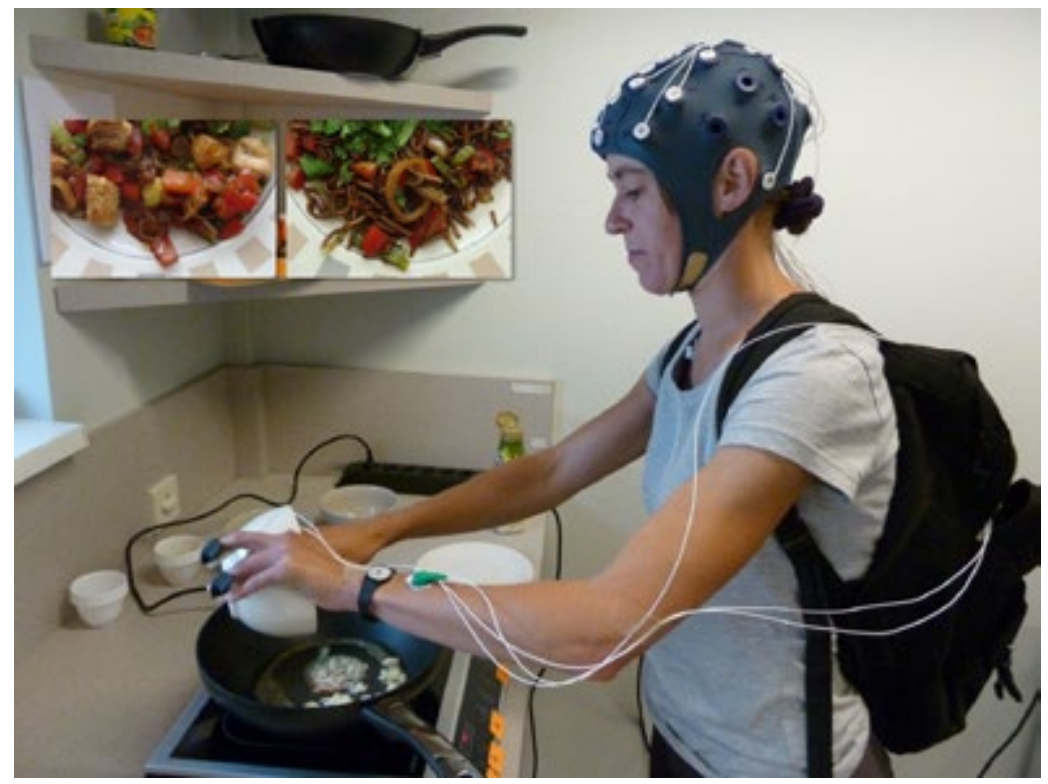

Figure 17. Implicit measures in a food context (Brouwer et al., 2017).

The dishes were expected to evoke 'approach', that is to say, pleasure, with the dish with chicken and 'avoidance', that is to say, disgust for the dish with mealworms. The real challenge in this study was to record physiological variables when participants were freely moving about while they were cooking. For example, any movement of the body immediately creates all kinds of brain signals, which makes it difficult to separate meaningful approach/avoidance signals from all the other signals being recorded by the EEG. By specifying a step-by-step script for the cooks and using state-of-the-art data-analysis techniques, we were able to filter out the large amount of noise in the data due to the movements as respondents were preparing 
the dishes. Interestingly, we found clear differences in neurophysiological patterns between the two emotional conditions. This was exciting as it opened up opportunities for the continuous monitoring of emotions in real-life settings at large scale. In a second study, subjects were cooking a stir-fry dish with small, more subtle differences in food stimuli, such as fresh herbs vs. dry herbs and premium packaging vs. basic packaging; differences that are too small to be picked up by rating scales, i.e., explicit measures. Unfortunately, these differences were also too small to be picked up by the implicit measures. The results to date are intriguing and warrant further investigation. We do not suggest replacing existing questionnaires but argue for optimal combinations of implicit measures such as heart rate and skin conductance and explicit measures such as choice making and questionnaires. The one supports and validates the other. For example, real time detection of emotionally salient events based on neurophysiological patterns can help to identify the best time slots for applying the questionnaires and thereby enable the development of more structured and precise tools for product design and development.

Although technologies in this area are developing rapidly, we have some way to go before we can (implicitly) measure emotional states and before we fully understand what is going on in the brain with respect to food reward and emotions. For the development and validation of implicit measures, research to date has deployed quite extreme differences in stimuli. It is important to explore more subtle, realistic stimuli and what happens when subjects move from 'Like a little' to 'Like a little bit more', as opposed to 'Like a lot' and 'Don't like this at all', as has currently been the focus in the literature. There is also the effect size issue to solve. We know what it means if someone says 'like a lot' vs. 'like extremely' but harder to say what a 5 (vs. 3 or 10) unit change in brain activation means, and whether these changes are meaningful in real-life contexts. My PhD student Maria Isabel Salazar Cobo, who is co-supervised by Dr. Gerry Jager and Prof. Kees de Graaf, will address some of these issues in the coming four years in a SENESCYT project that is supported by the government of Ecuador and Unilever R\&D Vlaardingen. She will be studying implicit measures of emotions that measure the dynamic interactions and experiences that might be experienced with 'real' foods in realistic eating contexts. And within the TKI Agri\&Food project 'Breaking habits for the better', we will explore the impact of repeated consumption on the acceptance of meat alternatives using implicit measures such as facial expression and heart rate as a new way to measure long-term acceptance in real-life settings. 


\section{Industry Sponsored Professorship}

Having spoken about my two focus areas of research, I now want to switch to the topic of industry-sponsored research. My professorship is a collaborative appointment between Wageningen University and Unilever R\&D Vlaardingen on a research area of mutual interest that both organisations have identified as innovative, purposeful and impactful. My chair falls under the full responsibility of Wageningen University and the standards and behaviours of the Netherlands Code of Conduct for Scientific Practice are the guiding principles for all activities within my research. In this way my research will comply to the highest level of scientific integrity and benefit science as a whole. I would like to emphasise that these guiding principles fully correspond to Unilever's Code for Responsible Innovation. I am honoured with this appointment and look forward to establishing a shared capability in the field of food reward and behaviour in which skills, expertise and facilities are jointly developed. I find this a clear win-win situation. It will facilitate combined developments of new scientific insights and applications. The university will have the opportunity to increase the economic value of science in this field and the company will gain more direct access into world-class scientific expertise and talent.

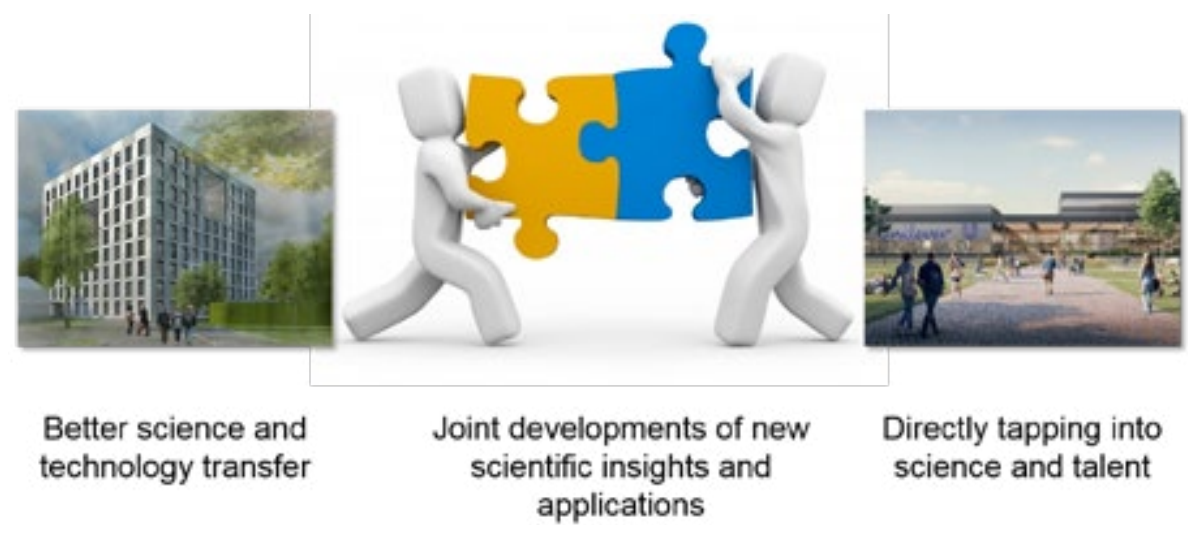

Figure 18. Strengthen the field of food reward and behaviour.

The research should also be attractive for students as it will give them the opportunity to learn about technology transfer to practical and innovative environments. In short, they will be more attractive to employers and they will benefit as a result. My personal experience is that practical challenges are fascinating and aspirational and can result in exciting fundamental scientific questions that require all of one's creativity and critical thinking to come to insightful solutions and useful applications. 


\section{Word of thanks}

This brings me to the end of my talk. In this talk, I explored matters of food reward and discussed why food rewards matter in relation to health. I can talk about this topic for many more hours and today I just gave you a few highlights and examples. Let me now turn to my word of thanks, the acknowledgements. There are many people with whom I have collaborated over the years, and others whose support and encouragement has been crucial. I would like to thank the Executive Board of Wageningen University \& Research and Unilever R\&D Vlaardingen for the opportunity to shape this research line within this special chair and the trust placed in me to fill in the chair well.

I would like to thank Prof. Wija van Staveren and Prof. Kees de Graaf, who formed the basis for my scientific career and on whose work I am now building upon. Wija, I don't know whether you remember that when I told you I took a position at Unilever that you said, "Now you are lost for science!". Soon you realized it was not that extreme. And the good news is, I am back again. Kees, we continued to collaborate throughout the years, in TIFN or NWO projects, meeting each other at conferences all over the world, and it has always been a pleasure. I was there at the start of your chair group Sensory Science and Eating Behaviour almost 10 years ago and I am impressed how quickly you have built such a strong group and put it on the international map in such a short time. I am proud to be part of your group.

I would like to thank my colleagues from Human Nutrition and Health, for the warm welcome I received, with special thanks for my colleagues from the Sensory Science and Eating Behaviour group for being such nice and enthusiastic colleagues. And not to forget the students, I truly love being surrounded by students from all over the world, with all the interest and questions they bring.

My colleagues from Unilever, I think it is great that you are here coming, from Vlaardingen to celebrate this special day with me. To Rob Hamer, thank you for all your tips and tricks and your experiences in guiding and supporting me in this trajectory of the special chair. I am glad that you are part of my curatorium. I would like to specifically thank my colleagues from Consumer Science and Sensory Science, from the past and current, who have given their support and their time in many of the experiments we have carried out together over the past eighteen years, thank you very much. 
To my family, and specifically my parents, you have always shown an interest in my work and have been supportive in what I do. I really appreciate that. The fact that you are all here, makes me really happy. And last but not least, Jan. Thank you for always being there, in good times and bad times. I think we form a unique team, at work and even more at home. And let me finish with thanking you, the audience, for your attention.

Ik heb gezegd. 


\section{Literature references}

1 National Center for Chronic Disease Prevention and Health Promotion, Division of Nutrition, Physical Activity, and Obesity, https://www.cdc.gov/obesity/data/ index.html (accessed at 27 September 2018).

2 International Food Information Council Foundation (2015). The 2015 Food \& Health Survey: Consumer attitudes toward food safety, nutrition and health. https://www.foodinsight.org/2015-food-health-survey-consumer-research.

3 Data MAT (2012). 2400 brands in 79 categories in 7 countries. ${ }^{\circ}$ Europanel.

4 Mischel, W., Shoda, Y., \& Rodriguez, M.L. (1989). Delay of gratification in children. Science, 244, 933-938.

5 Zandstra, E.H., Miyapuram, K.P., \& Tobler, P.N. (2013). Understanding consumer decisions using behavioural economics. In V.S. Chandrasekhar Pammi \& Narayanan Srinivasan (Eds.), Decision making: Neural and behavioural approaches. Progress in Brain Research, 202, 197-211.

6 Zandstra, E.H., Weegels, M.F., Van Spronsen, A.A., \& Klerk, M. (2004). Scoring or boring? Predicting boredom through repeated in-home consumption. Food Quality \& Preference, 15, 549-557.

7 Mela, D.J. (2000). Why do we like what we like? Journal of the Science of Food and Agriculture, 81, 10-16.

8 Bernstein, I.L., \& Webster, M.M. (1980). Learned taste aversions in humans. Physiology \& Behavior, 25, 363-366.

9 Booth, D.A., Mather, P., \& Fuller, J. (1982). Starch content of ordinary foods associatively conditions human appetite and satiation, indexed by intake and eating pleasantness of starch-paired flavours. Appetite, 3, 163-184.

10 Gould, N.J., Zandstra, E.H., \& Yeomans, M.R. (2018). Knowing too much: knowledge of energy content prevents liking change through flavour-nutrient associations. Quarterly Journal of Experimental Psychology, 71(9), 1939-1948.

11 Dijksterhuis, G.B. (2017). Hoe smaakt het? Nieuwe inzichten uit de wetenschap van proeven en ruiken. Uitgeverij Eburon, Delft, 147.

12 De Araujo, I.E., Rolls, E.T., Velazco, M.I., Margot, C., \& Cayeux, I. (2005). Cognitive modulation of olfactory processing. Neuron, 46, 671-679.

13 Plassmann, H., O'Doherty, J., Shiv, B., \& Rangel, A. (2008). Marketing actions can modulate neural representations of experienced pleasantness. Proceedings of the National Academy of Sciences of the United States of America, 105 (3), 1050-1054.

14 Zandstra, E.H., \& El-Deredy, W. (2011). Effects of energy conditioning on food preferences and choice. Appetite, 57, 45-49. 
15 Tijssen, I., Zandstra, E.H., de Graaf, C., \& Jager, G. (2017). Why a 'light' product package should not be light blue: Effects of package colour on perceived healthiness and attractiveness of sugar- and fat- reduced products. Food Quality $\mathcal{E}$ Preference, 59, 46-58.

16 Meiselman, H.L. (2006). The role of context in food choice, food acceptance and food consumption. In: The psychology of food choice. Shepherd, R., \& Raats, M. (Eds.), Wallingford, CABI, 179-200.

17 Edwards, J.S.A., Meiselman, H.L., Edwards, A., \& Lesher, L. (2003). The influence of eating location on the acceptability of identically prepared foods. Food Quality and Preference, 14, 647-652.

18 Hathaway, D., \& Simons, C.T. (2017). The impact of multiple immersion levels on data quality and panelist engagement for the evaluation of cookies under a preparation-based scenario. Food Quality E Preference, 57, 114-125.

19 Russell, J.A. (1980). A circumplex model of affect. Journal of Personality and Social Psychology, 39(6), 1161-1178.

20 Brouwer, A.-M., Hogervorst, M.A., Grootjen, M., Van Erp, J.B.F., \& Zandstra, E.H. (2017). Neurophysiological responses during cooking food associated with different emotions. Food Quality E Preference, 62, 307-316. 



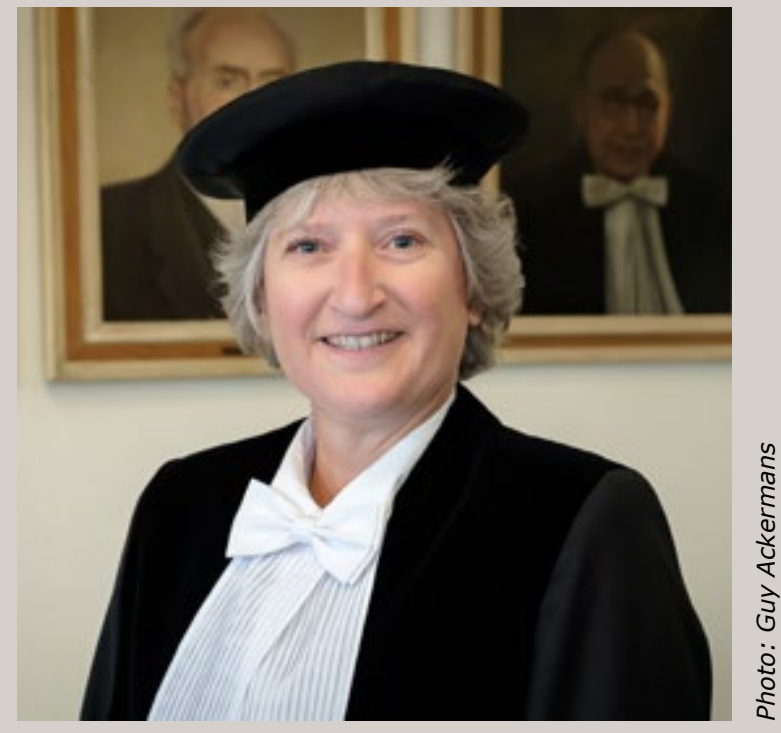

Prof. dr. Liesbeth Zandstra

'My research focuses on scientific challenges to find new ways to make the healthy choice the preferred choice. How can we motivate people to select healthier alternatives, i.e., products that are reduced in sugar, fat and salt? And that they don't do this only once, but over and over again, so that it becomes a habit. Consumer research consistently shows that taste is the key determinant that drives food choice. With taste, I also mean the smell, appearance and texture of foods - they are immediately rewarding. Health is only one of the many determinants that drives food choice, in addition to, for example, price, convenience and sustainability. The challenge here is that even though consumers know they need to eat healthily, they don't prioritize it at the point of purchase or consumption, as they don't perceive these effects as immediately and strongly as the taste of a product. I want to find out which other aspects are rewarding, to try and use these as a compensation for the lack of sugar, for example. Many factors play a role here, including emotions, social aspects and the physical context.' 